\title{
Prévision des précipitations par les modèles numériques de météorologie dynamique
}

\author{
Forecasting precipitation using dynamic meteorological \\ numerical models
}

\author{
D. Rousseau
}

Ingénieur en Chef de la Météorologie, EERM-GMD

\begin{abstract}
Les modèles numériques de météorologie dynamique simulent le comportement de l'atmosphère en résolvant les équations d'évolution des différentes variables caractérisant l'atmosphère; ces équations d'évolution sont établies en appliquant les lois de la dynamique, de la conservation de la matière, de la thermodynamique et de la physique. L'eau est une variable importante dans l'atmosphère et la prévision des précipitations est un des résultats fournis par ces modèles. Dans cet exposé nous présenterons les caractéristiques des modèles actuels de la Météorologie Nationale contribuant à la prévision des précipitations; nous donnerons également des éléments statistiques d'évaluation des prévisions quantitatives fournies automatiquement, sans adaptation statistique ou manuelle, par les modèles dynamiques depuis 1975 .
\end{abstract}

Description de l'eau dans un modèle de simulation atmosphérique

Un modèle de simulation décrit l'évolution des champs de vent, de pression, de température et de rapport de mélange de vapeur d'eau.

Pour les besoins du calcul numérique, ces différents champs sont discrétisés en un nombre fini de variables. Pour fixer les idées, les modèles actuellement en exploitation décrivent l'atmosphère par un maillage de points formant horizontalement un quadrillage de $150 \mathrm{~km}$ de côté, l'atmosphère étant divisée verticalement en dix couches.

Sur chaque point de ce maillage, sont déterminées les évolutions des variables qui sont donc représentatives d'un volume d'atmosphère de $150 \mathrm{~km} \times 150 \mathrm{~km} \times$ $1 \mathrm{~km}$ environ. On comprend qu'il faille distinguer, pour la prévision des précipitations, les phénomènes de grande échelle, qui peuvent être traités explicitement par le modèle (pluies de type continu) et ceux qui sont à une échelle inférieure à la maille de calcul (pluie de type convectif).

\section{Précipitations de grande échelle}

\section{Condensation}

La condensation se produit quand le rapport de mélange de vapeur d'eau $r$ atteint le rapport de mélange saturant $r_{w}$. Dans l'intégration numérique du modèle, on suppose dans un premier temps que la vapeur d'eau se conserve et donc que :

$$
\frac{d r}{d t}=0
$$

On obtient ainsi une première estimation $r^{\prime}$ du rapport de mélange à l'instant $t+\Delta t$.

Parallèlement, l'intégration de l'équation d'énergie fournit une première estimation $T^{\prime}$ de la température.

On compare alors $r^{\prime}$ au rapport de mélange saturant $r_{w}\left(T^{\prime}\right)$

Si $r^{\prime} \leqslant r_{w}$ il n'y a pas eu saturation et on conserve les valeurs obtenues.

Si $r^{\prime}>r_{w}$ on suppose qu'il y a eu condensation. On cherche alors de nouvelles valeurs $r^{\prime \prime}$ en $T^{\prime \prime}$ satisfaisant :

$$
\begin{gathered}
r^{\prime \prime}=r_{w}\left(T^{\prime \prime}\right) \\
C_{p}\left(T^{\prime \prime}-T^{\prime}\right)=L\left(r^{\prime}-r^{\prime \prime}\right)
\end{gathered}
$$

La première équation indique la saturation et la deuxième rend compte de l'échauffement de l'air correspondant au dégagement de la quantité de chaleur 
$L\left(r^{\prime}-r^{\prime \prime}\right)$ par transformation en liquide de la quantité $\left(r^{\prime}-r^{\prime \prime}\right)$.

Pour chaque couche du modèle, une quantité $M_{\ell}$ d'eau liquide est calculée.

Le schéma de précipitations exposé ci-dessous, redistribue cette eau liquide en tenant compte de son éventuelle évaporation lors de la précipitation.

\section{Schéma de précipitations}

On effectue le calcul en commençant par la couche supérieure du modèle et en procédant de haut en bas. Si éventuellement, une quantité $\Delta r=r^{\prime}-r^{\prime \prime}$ se trouve condensée dans une couche, cette eau liquide est transférée à la couche inférieure où la possibilité d'évaporation est examinée.

Deux cas peuvent se présenter :

- si cette couche est saturée et donc qu'une quantité d'eau s'y trouve également condensée, on l'ajoute au $\Delta r$ condensé dans la couche supérieure et le tout est transmis à la couche immédiatement inférieure ;

- si cette couche ne se trouve pas saturée après le calcul de l'évolution de la vapeur d'eau, la quantité $\Delta r$ est partiellement évaporée. Pour calculer cette évaporation, nous supposerons que les gouttes de précipitations peuvent être représentées par une loi de MarshallPalmer :

$$
N(D)=N_{0} e^{-\lambda D}
$$

$D$ est le diamètre d'une goutte, $N d D$ est le nombre de gouttes par unité de volume, de diamètre compris entre $D$ et $D+d D$.

Dans ces conditions, la masse d'eau liquide précipitante peut s'écrire :

$$
M_{\ell}=\frac{\pi \rho_{\ell} N_{0_{1}}}{\lambda^{4}}\left(\rho_{\ell}=10^{3} \mathrm{~kg} / \mathrm{m}^{3}\right)
$$

Le taux de variation de la masse $M_{i}$ d'une goutte d'eau en chute libre peut, selon Kessler [1], s'écrire :

$$
\frac{d M_{i}}{d t}=-2,24.10^{-2} D^{\Re / 5} \rho\left(r_{w}-r\right)
$$

Par intégration sur l'ensemble des gouttes, on obtient le taux d'évaporation de la précipitation :

$$
\frac{d M_{\ell}}{d t}=1,72.10^{-4} N_{0}^{7 / 20} \rho\left(r-r_{w}\right) M_{\ell}^{13 / 20}
$$

Si $R$ désigne l'intensité de précipitation, on aura :

$$
\frac{d R}{d z}=\frac{d M_{\ell}}{d t}
$$

Si PLH désigne la précipitation en haut d'une couche et $P L B$ la précipitation en bas, on obtient alors après intégration et en choisissant $N_{0}=10^{7} \mathrm{~m}^{-4}$

$$
\begin{aligned}
P L B^{0,4222}-P L H^{0,4222} & = \\
& -5.10^{-4} \sqrt{\sigma^{0.5777}} p_{s} \Delta \sigma\left(r_{w}-r\right)
\end{aligned}
$$

( $p_{s}$ désigne la pression au sol et $\sigma=p / p_{s}$ )
Si $P L B<0$ on pose $P L B=0$, la précipitation $P L H$ est entièrement évaporée dans la couche.

L'évaporation de $P L H-P L B$ accroit le rapport de vapeur d'eau $r$ de la couche et diminue sa température $T$ qui deviennent respectivement $r^{\prime}$ et $T^{\prime}$ tels que :

$$
r^{\prime}=r+(P L H-P L B) \frac{g \Delta t}{p_{s} \Delta \sigma}
$$

$$
T^{\prime}=T-\frac{L}{C_{p}}(P L H-P L B) \frac{g \Delta t}{p_{s} \Delta \sigma}
$$

Si les valeurs obtenues correspondent à une sursaturation $\left(r^{\prime}>r_{w}\left(T^{\prime}\right)\right)$, on recondense le surplus et on ajoute à $P L B$ la quantité d'eau ainsi obtenue.

En intégrant ce schéma de haut en bas, on obtient dans chaque couche les modifications au rapport de mélange de vapeur d'eau et à la température dues aux condensations et évaporations et les précipitations de grande échelle atteignant le sol.

\section{Précipitations convectives}

Les phénomènes convectifs sont matérialisés dans l'atmosphère par les nuages cumuliformes et par des précipitations sous formes d'averses.

Les dimensions horizontales de ces phénomènes sont trop faibles pour qu'ils soient explicitement décrit par les modèles de prévision actuels.

Cependant, on tente de paramétrer l'effet à plus grande échelle de ces phénomènes. Sans entrer dans le détail de la paramétrisation inspirée des travaux de Kuo [2], nous en indiquons ici le principe.

Sur chaque verticale, la possibilité d'existence d'une convection nuageuse est testée à chaque itération du modèle. Cette existence est présumée si deux conditions sont remplies :

- qu'il y ait dans les basses couches un apport positif d'humidité due à l'évaporation et à la convergence de vapeur d'eau;

- que l'atmosphère soit conditionnellement instable.

Si ces conditions sont satisfaites, le schéma de convection entre en jeu. Il permet de répartir en altitude l'énergie et l'humidité disponible dans les basses couches. $\mathrm{Au}$ cours de ce processus, le seuil de condensation peut être atteint à certains niveaux, ce qui donne lieu à l'occurrence d'une précipitation qualifiée de convective et comptabilisée pour la prévision.

\section{Facteurs influençant la prévision des précipitations}

La dynamique de l'atmosphère joue un rôle essentiel pour la prévision des précipitations et notamment l'évolution de la vitesse verticale de l'air : c'est en effet le soulèvement de l'air qui permet, par refroidissement adiabatique, d'atteindre, à rapport de mélange de vapeur d'eau constant, la température de condensation.

L'analyse initiale du champ de vapeur d'eau intervient pour la prévision à très courte échéance, mais la prévision à échéance plus éloignée est relativement insensible à ce facteur. Enfin, le modèle de simulation se doit de décrire aussi la source de vapeur d'eau de l'atmo- 
sphère, constituée par l'évaporation sur les océans et le sol. Pour des détails complémentaires concernant le modèle de prévision, le lecteur peut consulter les références [3] et [4].

\section{Vérification des prévisions quantitatives de précipitations}

Afin de pouvoir vérifier la qualité des prévisions de précipitations en temps quasi réel, le système de vérification mis au point s'appuie sur les observations aux stations synoptiques du réseau français (environ 150 stations). Ces observations permettent d'estimer la lame d'eau cumulée en 12 heures sur 25 grands bassins français par application de la méthode des polygones de Thiessen. La lame d'eau prévue sur chaque bassin est obtenue par la même méthode, à partir des valeurs prévues par le modèle en chaque point de grille. Les exemples de vérification qui sont présentés ci-dessous proviennent du modèle qui a précécé celui décrit plus haut (modèle à 5 niveaux sur une maille de $190 \mathrm{~km} \mathrm{x}$ $190 \mathrm{~km})[5]$.

\section{Diagrammes de dispersion et tableau de contingence}

A titre d'exemple la figure 1 donne pour le bassin de la Seine supérieure le diagramme de dispersion prévisionobservation pour la période de prévision $18 \mathrm{~h}-30 \mathrm{~h}$, Entre les droites indiquées sur la figure, se situent $90 \%$ des prévisions.

Une autre manière plus concise mais moins informative de présenter les résultats, consiste à utiliser un tableau de contingence; les précipitations sont divisées en classes. A titre d'exemple, le tableau I donne pour la prévision de 18 à $30 \mathrm{~h}$ les résultats obtenus sur l'ensemble des 25 bassins pendant 4 ans. Cinq classes ont été utilisées : précipitations inférieures à $0,1 \mathrm{~mm}$, comprises entre 0,1 et $1 \mathrm{~mm}$, comprises entre 1 et $5 \mathrm{~mm}$, comprises entre $5 \mathrm{~mm}$ et $10 \mathrm{~mm}$, supérieures à $10 \mathrm{~mm} / 12$ heures.

\section{Critère d'évaluation de la qualité de la prévision}

Un diagramme de dispersion tel celui présenté sur la figure 1 donne toute l'information sur la qualité de la

Tableau 1 - Table de contingence pour les lames d'eau estimées et prévues de 18 à $30 \mathrm{~h}$, calculées sur les 25 bassins d'avril 1975 à mars 1979 (31 705 prévisions contrôlées).

\begin{tabular}{|c|r|r|r|r|c|}
\hline \multicolumn{1}{|c|}{ Prévu } & \multicolumn{1}{c|}{0} & $0-1$ & $1-5$ & $5-10$ & $>10$ \\
\hline 0 & 14535 & 1636 & 926 & 164 & 127 \\
\hline $0-1$ & 3157 & 1335 & 1324 & 366 & 203 \\
\hline $1-5$ & 1787 & 1062 & 1696 & 697 & 532 \\
\hline $5-10$ & 282 & 181 & 436 & 260 & 281 \\
\hline$>10$ & 126 & 86 & 181 & 113 & 212 \\
\hline
\end{tabular}

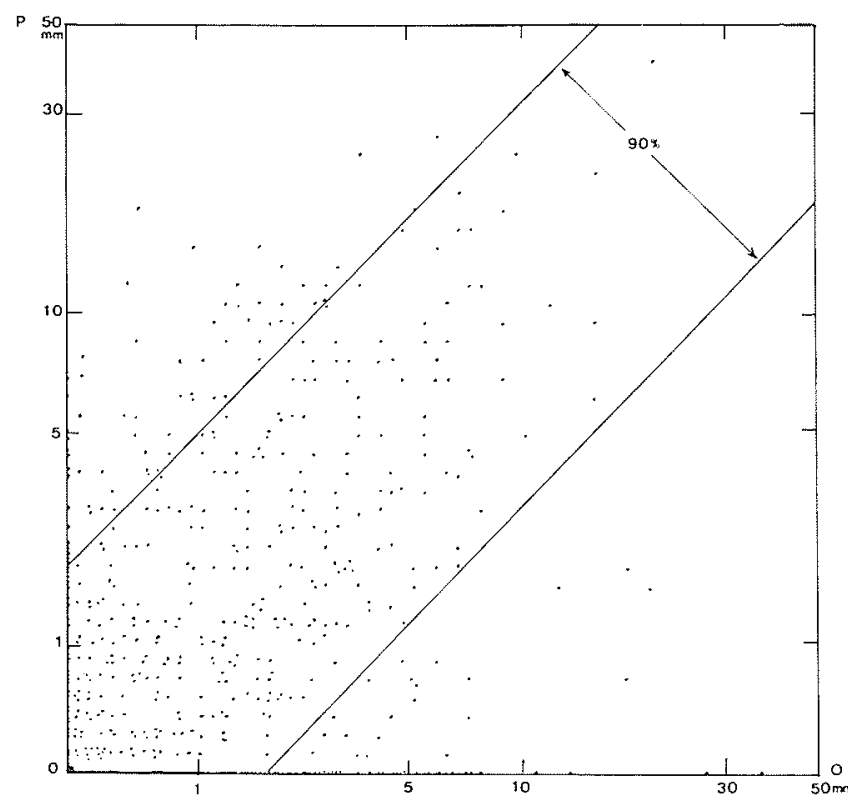

Figure 1 - Diagramme de dispersion Pluie observée- Pluie prévue de 18 à $30 \mathrm{~h}$ pour le bassin supérieur de la Seine d'avril 1975 à mars 1979 .

prévision fournie et permet à tout utilisateur d'évaluer l'utilité de celle-ci pour ses propres besoins. Il est cependant utile de disposer de critères plus synthétiques pour évaluer globalement les performances d'un système de prévision. Considérant un tableau de contingence du type :

\begin{tabular}{|c|c|c|}
\hline Observé & Prévue & Pluie $\leqslant S$ \\
\hline Pluie $\leqslant S$ & $a$ & $b$ \\
Pluie $>S$ & $c$ & $d$ \\
\hline
\end{tabular}

il est possible de définir un critère

$$
I=100 \times \frac{a d-\left(\frac{b+c}{2}\right)^{2}}{\left(a+\frac{b+c}{2}\right)\left(a+\frac{b+c}{2}\right)}
$$

qui prenne la valeur 0 pour une prévision ne faisant pas mieux qu'une prévision climatologique, donc sans valeur économique supplémentaire, et 100 pour une prévision parfaite [6].

En fixant différents seuils $S$, il est possible de juger du système de prévision selon l'intensité de précipitation considérée.

\section{Variation des performances selon l'échéance, la saison et l'intensité des précipitations}

Les indices $I$ ont été calculés bassin par bassin et sur l'ensemble des bassins pour différents seuils $S=0,1$ $1-5-10 \mathrm{~mm}$ pour les échéances du jour, de la nuit 


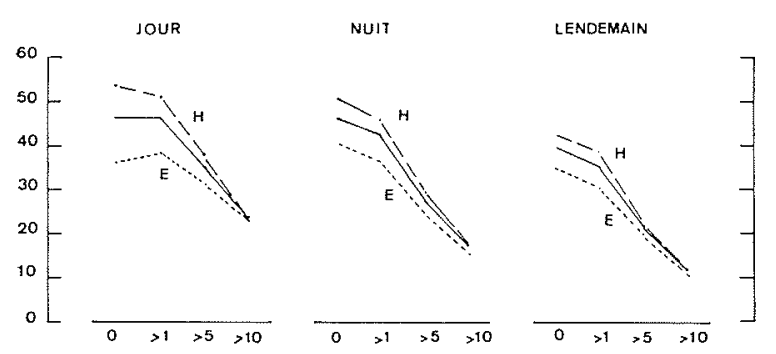

Figure 2 - Valeur des indices $I$ calculés sur l'ensemble des prévisions d'avril 1975 à mars 1979 et séparément sur l'hiver (H) et l'été (E) pour différents seuils et différentes échéances.

et du lendemain pour les mois d'octobre à mars (hiver) et d'avril à septembre (été) séparément. Ces résultats sont présentés pour l'ensemble des bassins sur la figure 2 .

On constate une diminution de l'indice moyen annuel de 46 à 40 entre la prévision d'occurence de précipitations mesurables pour le jour et celle pour le lendemain. Pour la prévision de fortes pluies $(>10 \mathrm{~mm}$ ), la diminution de la qualité est encore plus sensible puisqu'elle va de 23 à 11 .

Cette figure met en évidence également la différence entre la qualité de la prévision des pluies d'hiver et d'été : la qualité de la prévision pour la journée en été n'est pas supérieure par exemple à celle de la prévision pour le lendemain en hiver pour les pluies ne dépassant pas $1 \mathrm{~mm} / 12$ heures. Ces moins bonnes performances en été sont sans doute à relier à la nature plus convective des pluies d'été beaucoup moins bien traitées par le modèle.

On voit aussi sur ces figures que l'apport sur la climatologie s'affaiblit sensiblement pour les fortes précipitations. Il est à remarquer que pour les fortes précipitations, la distinction été-hiver s'estompe.

On peut penser que pour ces très fortes précipitations la convection et l'orographie jouent un grand rôle et que le modèle est déficient à cause de sa trop faible définition horizontale. Cependant, il convient de noter que, même pour les très fortes précipitations, l'information apportée par le modèle est significativement positive.

\section{Conclusion}

Les modèles de prévision dynamique permettent d'effectuer une prévision de précipitations. La façon de représenter le phénomène dans les modèles d'exploitation actuels a été décrite. On a fait une estimation détaillée des performances du modèle seul pour la prévision des lames d'eau sur la France divisée en 25 grands bassins.

Des techniques d'adaptation statistiques ou manuelles peuvent être mises en ouvre pour améliorer les performances du modèle de prévision dynamique, faisant usage de l'information apportée par le modèle et de tous les autres éléments (d'observation surtout) disponibles. C'est pourquoi ces statistiques ne doivent pas être interprêtées comme l'état actuel de l'art pour la prévision des précipitations, mais plutôt comme une limite inférieure de la qualité de la prévision assurée automatiquement par le modèle dynamique.

Dans le cadre des prévisions du modèle, des améliorations sont attendues du développement général des modèles (meilleure définition horizontale et verticale, meilleures paramétrisations) et d'une meilleure information initiale (données satellites et radar par exemple) pouvant surtout bénéficier à la très courte échéance.

\section{Références}

[1] KESSLER E. - On the distribution and continuity of water substance in atmospheric circulations. Meteorological Monographs, 1969 , Volume $10 \mathrm{n}^{\circ} 32$.

[2] KUO. - On the formation and intensification of tropical cyclones through latent heat release by cumulus convection, J. Atmos. Sci., 1965, 22.

[3] COIFFIER J. et al. - Projet Amethyste Cahier $\mathrm{n}^{\circ} 1-L e$ modele de prévision en points de grille - Partie dynamique - Note technique de l'EERM n ${ }^{\circ} 36,1979$.

[4] LEPAS J. et al. - Projet Amethyste Cahier n ${ }^{\circ} 2-L e$ modèle de prévision - Partie physique - Note technique de l'EERM n $37,1979$.

[5] ROUSSEAU D. - Les modèles de la prévision numérique - La Météorologie. VI ${ }^{\mathrm{e}}$ série, 1976, $\mathrm{n}^{\mathrm{N}} 7$.

[6] ROUSSEAU D. - Contrôle des prévisions. Partie II - Vérification des prévisions de l'occurrence d'un phénomère. Application aux prévisions de précipitation. Note technique de l'EERM, n 80, 1980. 


\section{Discussion}

Président : M. J. JACQUET

Le Président. - Merci M. ROUSSEAU de nous avoir démonté le mécanisme de ce modèle numérique et de nous avoir montré les perspectives ouvertes par le maillage spatial très fin à partir duquel vous pensez pouvoir travailler maintenant. Ce projet est directement lié aux disponibilités de nouveaux moyens de calcul, je suppose. Quels sont vos espoirs de ce côté-là ?

M. ROUSSEAU. - Dans un avenir proche nous disposerons des calculateurs du centre européen. Actuellement, nous travaillons sur l'ordinateur de la Météorologie Nationale en essayant de perturber le moins possible les travaux de routine.

Le Président. - Comment voyez-vous, M. DUBAND, l'utilisation des prévisions dans l'hypothèse où on vous les fournit avec la grille présentée par M. ROUSSEAU ? Pensez-vous pouvoir les utiliser directement ou procèderez-vous encore à une adap tation locale, par corrélation par exemple ?

M. DUBAND. - Pour faire des corrélations, il faut avoir des données historiques. Or, c'est un modèle qui est récent ; il n'y aura pas d'échantillons importants.

Mais l'idée de M. ROUSSEAU, qui consisterait à associer cette pluie prévue macro-spatialement, à des zones beaucoup plus réduites est peut-être une idée à creuser; il faut cependant dissocier ce modèle des méthodes actuelles : c'est une autre approche.

M. ROUSSEAU. - La maille de $35 \mathrm{~km} \times 35 \mathrm{~km}$ est évidemment assez large et il sera toujours nécessaire de travailler à une échelle plus fine et de trouver des modèles d'adaptation. Dès qu'on a un historique d'une ou deux années, on peut envisager une utilisation de données très élaborées. Cependant une difficulté supplémentaire tient à l'évolution du modèle qui est constamment perfectionné si bien que ses sorties ne sont pas stationnaires.

Je crois que les deux modèles (Météo et EDF) sont différents et resteront en concurrence.

Le Président. - Les résultats du modèle sont moins bons pour les précipitations importantes. Avec le modèle EDF c'est le contraire, et ceci confirme bien la différence des approches.

M. ROUSSEAU. - Les résultats du modèle de la Météorologie sont malgré tout très "cadrés" par rapport à la rareté du phénomène. Pour les pluies supéricures à $5 \mathrm{~mm}$ en 12 hcures, avoir une certaine prévisibilité n'est pas si mal. Pour les précipitations supérieures à $30 \mathrm{~mm}$ en 72 heures, évidemment les prévisions sont plus mauvaises, mais non totalement négatives.

Le Président. - Qu'a donné le modèle utilisé en prévision pour les averses génératrices des crues de la Loire en Septembre 1980 ?

M. ROUSSEAU. - C'est un facteur 4 de différence. Il aurait fallu un modèle à maille plus fine.

M. GUILLOT. - La déception habituelle du non-spécialiste est de constater que la lourdeur des modèles atmosphériques est telle qu'il n'est pas possible de les ajuster, mais seulement de constater après coup leur score sur quelques saisons. Ne croyez-vous pas possible, vous qui connaissez bien la structure de vos modèles, de les remplacer par des "modèles simplifiés équivalents" gagnant un ou plusieurs ordres de grandeur du temps de calcul, et que vous pourriez cette fois ajuster systématiquement sur des séquences de situations observées?

M. ROUSSEAU. - Je pense que c'est une très bonne idée. Mais en ce domaine, on en est actuellement au stade de l'artisanat. Il y a quand même eu des itérations puisque le modèle Améthyste actuel est la $10^{\mathrm{e}}$ version d'un modèle qui a été sélectionné sur 20 cas. Cependant l'idée d'un modèle simplifié qu'on pourrait faire passer simultanément en plusieurs versions est certainement une bonne utilisation du calculateur, je suis parfaitement d'accord avec vous.

Il faut dire que jusqu'à présent ce sont les erreurs de troncatures numériques qui ont été prépondérantes. Les progrès étaient assurés en utilisant au maximum les possibilités du calculateur. Nous en sommes peut être actuellement au point où l'on va se restreindre volontairement pour pouvoir passer beaucoup de versions et faire de la statistique sur les modèles dy namiques.

M. THIERY. - Le modèle fait-il intervenir des paramètres purement physiques comme la constante de Stefan BOLZMAN, ou utilise-t-il des paramètres d'échange ajustés par tatonnement ou susceptibles d'être ajustés, comme la transmissivité pour les nappes alluviales par exemple.

M. ROUSSEAU. - Le modèle comporte plusieurs partics: une partie dynamique avec des équations intouchables et une autre partie paramètrée. Il y a différents stades; on peut adapter certains schémas sur des modèles unidimensionnels. Par contre, il $y$ a des facteurs qu'on ne peut adapter qu'à partir du moment où on a mis le tridimensionnel en route : coefficients de diffusion, de frottement... qui sont fonction de l'échelle et des autres paramétrisations. Ce problème d'optimisation des paramètres à l'intérieur d'un modèle est important, mais on cherche des raisons extéricures à la prévision pour les ajuster et donc des raisons théoriques. L'ajustement des données météorologiques est malgré tout quelque chose de délicat : c'est l'ajustement des champs.

M.S. MARTIN. - Comment avez-vous pris en compte l'effet de persistance dans vos modèles de prévision comparatifs ? Est-ce en reconduisant l'état de la veille ou en utilisant des modèles markoviens?

M. ROUSSEAU. - Il s'agissait des réalisations de la veille c'est-à-dire du cumul des précipitations en 12 heures, entre 18 heures la veille et 6 heures le lendemain, pour la prévision entre 6 heures et 18 heures.

Le Président. - Je remercie encore M. ROUSSEAU. 


\section{Abstract \\ Forecasting precipitation using dynamic meteorological numerical models}

Dynamic numerical meteorological models simulate atmospheric conditions by solving the equations for the trend of the different variables describing the atmosphere. Water is a major variable in the atmosphere; precipitation is one of the results yielded by these models.

The forecast of precipitation involves the identification of large scale phenomena which can be explicitly processed by the model (with a resolution of the order of $150 \times 150 \mathrm{~km}$ ) and smaller scale phenomena which are below the calculation range (convective type rains).

Explicit processing takes into account the water vapour transfer, condensation and the resulting heat discharge. A simple chart of precipitation, assuming a Marshall-Palmer-type distribution of rainfall, allows the volume of water reaching the ground to be calculated, taking account of the evaporation of raindrops in non-saturated layers.

Convective effects are parametered in a chart which distributes thermal energy and humiduty as a function of altitude when the model indicates the existence of cloud convention, following two criteria: conditionally unstable atmosphere and positive water vapour addition at the base by convergence and evaporation.

Rainfall forecasts have been made for France over 25 basins for 12 hour stretches during the day, at night and the following day. A verification criterion:

$$
I=\frac{100 \mathrm{ad}-\left(\frac{b+c}{2}\right)^{2}}{\left(a+\frac{b+c}{2}\right)\left(d+\frac{b+c}{2}\right)}
$$

has been defined, in which $a, b, c$ and $d$ are the coefficient of the contingency table of rain observed and forecast exceeding a certain threshold. The value 100 of the index corresponds to a perfect forecast, and the value 0 describes a meteorological forecast of no value.

Quality deteriorates the further forward the forecasting and the greater the rate of precipitation. Nevertheless, the model is always helpful. In addition, it can be seen that summer rain is not forecast as well as winter rain, in particular for moderate and low rainfall.

These criteria relating to direct forecasts from the model can be used as a basis for assessing the improvements which can possibly be obtained through dynamic or statistical forecasts through local adjustements. 